\title{
Special Issue on Current Developments and State-of-the-art in Unmanned Aircraft Systems
}

\author{
Kimon P. Valavanis
}

Published online: 29 September 2012

(C) Springer Science+Business Media Dordrecht 2012

Dear Colleagues,

This double Volume ( 8 issues total), which is split in Volume 69 and 70, is devoted to the state-of-the-art and recent advances in Unmanned Aircraft Systems (UAS). The area of UAS has seen unprecedented levels of growth over the last decade, and it is also expected to be one of the most dominant areas of research and development in the future.

Although the main focus of UAS applications was in military domains, we now see a shift of UAS use in civilian/public domains. However, it will be long before UAS are extensively utilized in civilian domains, but their importance in applications related to earth science, environment/pollution monitoring, land management, civil infrastructure health management, public security, fire detection, emergency response, search and rescue, etc., is obvious.

Despite the tremendous progress in UAS, there are still major challenges and obstacles to be

K. P. Valavanis $(\varangle)$

Department of Electrical and Computer Engineering, School of Engineering and Computer Science,

University of Denver, Denver, CO 80208, USA

e-mail: kimon.valavanis@du.edu,

Kimon.Valavanis@exch.du.edu overcome, which relate, among other things, to: navigation, control and communication issues; levels of autonomy; fault-tolerance; sensors; payloads, range and endurance, and, integration of UAS into the national airspace.

This double Volume aims at discussing some of the current challenges in UAS by publishing peer reviewed, archive and original papers from research groups all over the world. The papers have been divided in the following areas:

1. UAS operations, regulations and airworthiness

2. Energy efficient UAV systems and UAS management

3. UAV design, modeling and validation

4. UAV control

5. UAV mission and path planning

6. UAV tracking

7. UAV vision systems and vision-based navigation

8. Quadrotor UAVs

9. Micro Air-Vehicles (MAVs)

10. UAV formations and swarms

11. Cooperative UAVs

12. UAS testbeds

13. UAV Applications.

The list of topics is by no means exclusive and complete, but it does provide an indication of the wide spectrum of problems scientists, researchers and engineers work on. An important part of 
the double is conducted research in cooperative UAVs and UAV formations, which hints a move from a single UAV to multiple UAVs. Regardless, the list of topics is very wide and diverse, and the Applications section papers give the reader nothing but an idea of where UAVs may be used.

Volume 69 includes topics 1-7 and Volume 70 includes topics 8-13.
We hope that the information provided in this double Volume is helpful and that it will make the readers aware of the level of maturity of UAS related accomplishments.

Kimon P. Valavanis

Guest Editor 